\title{
Calculating the movement of MRI coils, and minimizing their noise
}

\author{
L. K. Forbes ${ }^{1} \quad$ M. A. Brideson ${ }^{2} \quad$ S. Crozier $^{3}$ \\ P. T. While ${ }^{4}$
}

(Received 26 July 2007; revised 7 September 2007)

\begin{abstract}
The design of gradient coils within Magnetic Resonance Imaging equipment is considered. These coils produce linear magnetic fields in each of the three orthogonal directions in physical space. In addition, they are turned on and off repeatedly to enhance the clarity of the image, but this produces a great deal of noise within the coil, as its shape distorts under the influence of Lorentz forces. We present a method for calculating the movement of the coil in the background magnetic field, and estimating the consequent noise levels. This involves solving for the current density in the coil coupled with equations for its elastic deformation, along with acoustic equations for the pressure in the surrounding air. Winding patterns are designed to minimize the noise produced by the Magnetic Resonance Imaging coil.
\end{abstract}

See http://anziamj.austms.org.au/ojs/index.php/ANZIAMJ/article/view/319 for this article, (c) Austral. Mathematical Soc. 2007. Published September 15, 2007. ISSN 1446-8735 


\section{Introduction}

Magnetic Resonance Imaging (MRI) equipment is an important diagnostic imaging tool in many hospitals. In conventional systems the patient lies within the cylindrical bore of the MRI device, and there are electromagnetic coils of various types positioned around its cylindrical surface. One set of windings is responsible for producing a strong homogeneous stationary magnetic field, that aligns the magnetic moments of hydrogen nuclei in the body. Gradient coils are also present, and their function is to add a magnetic field that varies linearly in each of the three orthogonal directions, so producing a unique field at each point. Finally, there are radio frequency coils that serve the dual role of exciting the aligned protons from one quantized state to a higher one, and then receiving the radio frequency signal emitted as the protons return to their lower energy state when the signal is removed. Because the frequency at which the signal is absorbed varies linearly with the strength of the background field, the physical location of a received signal is known in advance due to the effect of the gradient coils, which are therefore frequency encoding devices. Jin [10], Vlaardingerbroeck and den Boer [16] and Haacke et al. [9] describe the operation of MRI equipment.

The design of winding patterns for MRI coils is a classical inverse problem, in the sense that the magnetic field, normally an output quantity, is desired to be specified in advance over some region within the coil (referred to as the diameter of the sensitive volume or DSV). The shape of the coil winding pattern is then to be determined. This is known to be an ill-conditioned problem, since many different winding patterns could give very similar magnetic fields over the DSV.

Much has been written on the design of MRI coils, and there are various methods in existence for solving this ill-conditioned problem. Turner [15] developed an approach based on Fourier transforms, since these provide an immediate solution to the difficulty of ill-conditioning, although at the cost of requiring coils that are notionally infinite in length. An optimization 
technique based on simulated annealing was developed by Crozier and Doddrell [4] and solved for the current in discrete coils, along with their physical location. More recently, Forbes and Crozier [6, 7, 8] developed a method that is essentially an extension of Turner's target field approach [15], but accounts for finite length coils exactly. This technique treats the coil windings on the cylinder as equivalent to a continuous current sheet on its surface, and reduces the problem to a first kind integral equation for the current density there. This equation is too ill-conditioned to be solved directly, but it can be solved in a weaker, least squared, sense by minimizing the square of its error while simultaneously minimizing some other quantity, using a Lagrange multiplier approach. This technique is known as Tikhonov regularization, and is discussed by Delves and Mohamed [5]. The choice of the extra (regularizing) function to be minimized along with the error in the integral equation is up to the designer, and Forbes and Crozier [8] used a function that effectively minimized the curvature in the coil windings. In this article we instead minimize the overall coil deflection in order to reduce noise.

The noise generated within a coil has been studied extensively. Chapman and Mansfield [3] and Mansfield et al. [11] used the concept of balancing the Lorentz forces on each conductor in the coil with a conductor carrying an equal but opposite current. They showed that noise reduction of about $10 \mathrm{~dB}$ was possible with this technique, although very high levels of about $130 \mathrm{~dB}$ may nevertheless occur. Finite element techniques were used by Mechefske et al. [12] to model the deformation of a gradient coil in response to Lorentz forces, and they showed that their approach gave good agreement with experiments, and produced noise levels between $120 \mathrm{~dB}$ and $140 \mathrm{~dB}$. More recently, Shao and Mechefske $[13,14]$ used analytical methods to study noise generation in cylindrical ducts. Here, we develop simplified equations for modelling coil deflection and the noise it generates, and use analytical methods to minimize the overall deflection directly as part of the optimization strategy. 


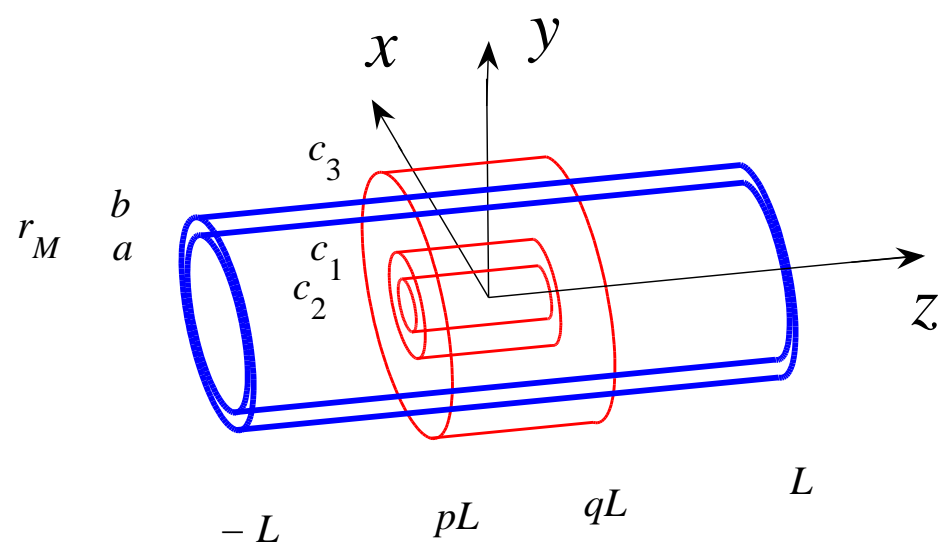

Figure 1: A sketch of the cylindrical coil and target zones.

\section{Mathematical model}

Consider a cylindrical duct of length $2 L$. The $z$-axis of a Cartesian coordinate system is located along its axis, so that the coil lies in the interval $-L<z<$ $L$. The inner radius of the coil is $a$, and the primary windings are placed on this surface. Its outer radius is $b=a+h$, in which the coil thickness is $h$, and shielding windings are located at this radius. For later reference, a midline radius $r_{M}=a+h / 2$ is also defined, and this coil geometry is illustrated in Figure 1. The material properties of the coil are its Young's modulus $E$ and its Poisson's ratio $\nu$. 
For simplicity, the spherical DSV is replaced here with a cylindrical region of interest positioned co-axially with the coil but located asymmetrically with respect to its length, over some interval $p L<z<q L$ with $-1<p<q<1$. The desired target magnetic field is specified over this cylinder, which is taken to have radius $c_{1}$ as shown in Figure 1. For increased accuracy, the field is also prescribed on an inner target cylinder of radius $c_{2}\left(<c_{1}\right)$. When a shield winding is also present on the outer surface $r=b$ of the coil, then a third target cylinder of radius $c_{3}(>b)$ is also employed, and the intention is to make the magnetic field drop to zero on that outer surface. It is convenient to make use of cylindrical polar coordinates $(r, \theta, z)$ defined according to $x=r \cos \theta$ and $y=r \sin \theta$, and these will be used from now on.

The component of the magnetic field $\mathbf{H}$ that is of the greatest interest is the axial component $H_{Z}$ directed along the $z$-axis. According to the BiotSavart law [9, p.828], this is

$$
\begin{aligned}
& H_{Z}(r, \theta, z)= \\
& -\frac{a}{2 \pi} \int_{-L}^{L} \int_{0}^{2 \pi} \frac{\left[r \cos \left(\theta^{\prime}-\theta\right)-a\right] j_{P \theta}\left(\theta^{\prime}, z^{\prime}\right)}{\left[a^{2}+r^{2}-2 a r \cos \left(\theta^{\prime}-\theta\right)+\left(z^{\prime}-z\right)^{2}\right]^{3 / 2}} d \theta^{\prime} d z^{\prime} \\
& -\frac{b}{2 \pi} \int_{-L}^{L} \int_{0}^{2 \pi} \frac{\left[r \cos \left(\theta^{\prime}-\theta\right)-b\right] j_{S \theta}\left(\theta^{\prime}, z^{\prime}\right)}{\left[b^{2}+r^{2}-2 b r \cos \left(\theta^{\prime}-\theta\right)+\left(z^{\prime}-z\right)^{2}\right]^{3 / 2}} d \theta^{\prime} d z^{\prime} .
\end{aligned}
$$

Here, the source points $\left(a, \theta^{\prime}, z^{\prime}\right)$ and $\left(b, \theta^{\prime}, z^{\prime}\right)$ lie on the inner and outer surfaces $r=a$ and $r=b$ of the coil, and the field point is $(r, \theta, z)$. We assume in the derivation of equation (1) that current flows on both sides of the conducting elements on each surface.

The current density $\mathbf{j}_{\mathbf{P}}(\mathrm{A} / \mathrm{m})$ on the primary $r=a$ may be expressed as $\mathbf{j}_{\mathbf{P}}=j_{P \theta} \mathbf{e}_{\theta}+j_{P Z} \mathbf{e}_{\mathbf{z}}$ with components $j_{P \theta}$ and $j_{P Z}$ in the azimuthal and axial directions, respectively. In steady state operation, the continuity equation is $\operatorname{div} \mathbf{j}_{\mathbf{P}}=0$, and it follows at once from a vector identity that

$$
j_{P \theta}=\frac{\partial \psi_{P}}{\partial z}, \quad j_{P Z}=-\frac{1}{a} \frac{\partial \psi_{P}}{\partial \theta},
$$


in which $\psi_{P}(\theta, z)$ is a streamfunction on the primary coil. Similar equations to (2) apply on the shield coil at $r=b$, and involve current density components $j_{S \theta}$ and $j_{S Z}$ and a streamfunction $\psi_{S}(\theta, z)$ on that surface. As the axial current density components $j_{P Z}$ and $j_{S Z}$ are required to be zero at each end $z= \pm L$ of the coil, it follows that the streamfunction on the primary coil must take the form

$$
\begin{aligned}
& \psi_{P}(\theta, z)=-\sum_{n=1}^{N} \frac{2 L}{n \pi} P_{0 n}^{P} \cos \left(\frac{n \pi(z+L)}{2 L}\right) \\
& +\sum_{m=1}^{M} \sum_{n=1}^{N} \frac{2 L}{n \pi}\left[P_{m n}^{P} \cos m \theta+Q_{m n}^{P} \sin m \theta\right] \sin \left[\frac{n \pi(z+L)}{2 L}\right] .
\end{aligned}
$$

The integers $M$ and $N$ in equation (3) are chosen to be suitably large. A similar equation to (3) exists for the streamfunction $\psi_{S}$ on the shields, except that the two sets of primary coefficients $P_{m n}^{P}$ and $Q_{m n}^{P}$ are replaced with corresponding shield coefficients $P_{m n}^{S}, Q_{m n}^{S}$. As yet, these four sets of coefficients are unknown, and an algorithm for determining them will be outlined in section 3. Equally spaced contours of each streamfunction then correspond to actual winding patterns on each coil, as shown by Brideson et al. [2].

The deflection of the coil is represented by the vector $\mathbf{u}=u_{R} \mathbf{e}_{\mathbf{r}}+u_{\theta} \mathbf{e}_{\theta}+$ $u_{Z} \mathbf{e}_{\mathbf{z}}$, and the equations of linearized elasticity at steady state become

$$
\frac{1}{h}(\mathbf{j} \times \mathbf{B})+(\Lambda+G) \nabla(\nabla \cdot \mathbf{u})+G \nabla^{2} \mathbf{u}=\mathbf{0} .
$$

In this equation (4), $\Lambda=\nu E /[(1+\nu)(1-2 \nu)]$ and $G=E /[2(1+\nu)]$ are Lamé coefficients involving the Young's modulus $E$ and Poisson's ratio $\nu$ of the coil material. The vectors $\mathbf{j}$ and $\mathbf{B}$ represent the total current density on the coil and the magnetic induction field, and their vector product in equation (4) is the Lorentz force on the coil. This equation can be derived using results by Boresi and Chong [1]. It is now assumed that the radial deflection component $u_{R}$ is the dominant one, so that the simple scalar differential 
equation

$$
\frac{1}{r_{M}^{2}} \frac{\partial^{2} u_{R}}{\partial \theta^{2}}+\frac{\partial^{2} u_{R}}{\partial z^{2}}-\frac{u_{R}}{r_{M}^{2}}=-\Delta_{0}\left(j_{P \theta}+j_{S \theta}\right)
$$

is obtained, in which the constant $\Delta_{0}=4(1+\nu) B_{Z 0} /(E h)$, and $B_{Z 0}$ is the $z$-component of the stationary magnetic induction field produced by the 'permanent' electromagnets in the MRI device. Equation (5) is assumed to hold on the coil centre plane shown in Figure 1. The current density components $j_{P \theta}$ and $j_{S \theta}$ are computed from equations (2) and (3).

The air pressure $p_{A}$ within the coil $0<r<a$ is calculated using the standard wave equation,

$$
\frac{\partial^{2} p_{A}}{\partial t^{2}}=c_{A}^{2} \nabla^{2} p_{A}=c_{A}^{2}\left[\frac{1}{r} \frac{\partial}{\partial r}\left(r \frac{\partial p_{A}}{\partial r}\right)+\frac{1}{r^{2}} \frac{\partial^{2} p_{A}}{\partial \theta^{2}}+\frac{\partial^{2} p_{A}}{\partial z^{2}}\right]
$$

that comes from linearized acoustic theory. Here, the wave speed $c_{A}$ has a known value (and may also be computed using the ideal gas law). We assume that the coil moves quasi-statically; that is, its motion due to the changing current in the coil windings is slow by comparison with the time taken for elastic waves to propagate in the material. As a consequence, the coil is considered to pass through a sequence of steady states as it deflects under the effect of the Lorentz forcing. We assume that the radial component of the deflection of the coil varies with time $t$ according to some switching function $f_{S}(t)$, so that the boundary condition for equation (6) on the inside wall of the coil is

$$
\frac{\partial p_{A}}{\partial r}=-\rho_{A 0} f_{S}^{\prime \prime}(t) u_{R}(\theta, z) \quad \text { on } r=a
$$

and initial conditions for the pressure in the coil are taken simply to be $p_{A}=0$ and $\partial p_{A} / \partial t=0$ at $t=0$. The constant $\rho_{A 0}$ in equation (7) represents the mean air density in the coil. The switching function $f_{S}(t)$ describes how the coil walls move with time, and so it determines the air pressure inside the primary. It is assumed to have a continuous second derivative for $t>0$. Once the air pressure has been calculated from equations (6) and (7) and 
using the coil deflection function $u_{R}$ obtained from equation (5), the sound pressure level in decibels is

$$
\mathrm{SPL}=20 \log _{10}\left(\left|p_{A}\right| / p_{\mathrm{ref}}\right) .
$$

The reference pressure $p_{\text {ref }}=2 \times 10^{-5} \mathrm{~N} / \mathrm{m}^{2}$.

\section{Outline of solution technique}

The Biot-Savart law (1) is normally used to calculate the magnetic field $H_{Z}$ for given current densities in the coil. Here, however, we solve the inverse problem, in which desired 'target' magnetic fields $H_{T Z}^{(1)}, H_{T Z}^{(2)}$ and $H_{T Z}^{(3)}=0$ are specified in advance at the three target radii $c_{1}, c_{2}$ and $c_{3}$ shown in Figure 1. Equation (1) is then solved as an integral equation for the two current density components $j_{P \theta}$ and $j_{S \theta}$. However, this is a highly ill-conditioned problem, and cannot be solved directly. Instead, we minimize the total regularized residual error

$$
R=E_{1}+E_{2}+E_{3}+\lambda_{1} F_{1}+\lambda_{P} F_{P}+\lambda_{S} F_{S},
$$

in which the three functionals

$$
E_{k}=\iint\left[H_{T Z}^{(k)}-H_{Z}\left(c_{k}, \theta, z\right)\right]^{2} d S, \quad k=1,2,3,
$$

are the total squared differences between the target field and the calculated field, with the integrals evaluated over the three cylindrical target surfaces shown in Figure 1. The three constants $\lambda_{1}, \lambda_{P}$ and $\lambda_{S}$ are regularizing parameters. They play a role similar to that of Lagrange multipliers in constrained minimization problems, except that here their value is not known in advance, and must be determined empirically. The first penalty function in equation (9) is chosen to be

$$
F_{1}=\int_{-L}^{L} \int_{-\pi}^{\pi} u_{R}^{2}(\theta, z) d \theta d z
$$


and represents the total squared coil deflection. The other two quantities are smoothness penalty terms

$$
F_{P}=\iint_{r=a}\left\|\nabla^{2} \psi_{P}\right\|^{2} d S, \quad F_{S}=\iint_{r=b}\left\|\nabla^{2} \psi_{S}\right\|^{2} d S,
$$

evaluated over the primary and the shield, respectively. Minimizing these quantities produces coil winding patterns with minimal curvature.

The residual error (9) is minimized with respect to the unknown Fourier coefficients by requiring that

$$
\begin{aligned}
& \frac{\partial R}{\partial P_{j k}^{P}}=0, \quad \frac{\partial R}{\partial P_{j k}^{S}}=0, \quad j=1, \ldots, M, \quad k=0,1, \ldots, N, \\
& \frac{\partial R}{\partial Q_{j k}^{P}}=0, \quad \frac{\partial R}{\partial Q_{j k}^{S}}=0, \quad j=1, \ldots, M, \quad k=1, \ldots, N, .
\end{aligned}
$$

This system (13) is a linear matrix set of equations for the unknown coefficients $P_{m n}^{P}, Q_{m n}^{P}$ and $P_{m n}^{S}, Q_{m n}^{S}$ and may be solved in a straightforward manner. There are computational efficiencies available that allow the block structure of the system to be exploited, giving savings in computer time, but these are not be detailed here. We make use of closed form solutions for the radial coil deflection function $u_{R}$ in equation (5) and the air pressure $p_{A}$ in equations (6)-(7) (obtained using Laplace transforms). This gives additional savings in computer time.

\section{Presentation of results}

Results are illustrated briefly in this section, for a model coil of half-length $L=0.5 \mathrm{~m}$ and inner radius $a=0.2 \mathrm{~m}$. Its wall thickness is taken to be $h=0.015 \mathrm{~m}(15 \mathrm{~mm})$, and its Young's modulus and Poisson's ratio are $E=$ $1.3 \times 10^{10} \mathrm{~N} / \mathrm{m}^{2}$ and $\nu=0.2$, respectively. The background constant axial 
field is taken to be $B_{Z 0}=2$ Tesla and the gradient coil is assumed to produce a maximum magnetic field $H_{\max }=1.59 \times 10^{4} \mathrm{~A} / \mathrm{m}$ (corresponding to an induction field of 0.02 Tesla). The inner target region (illustrated in Figure 1) is very asymmetrically positioned, with parameters $p=-0.7$ and $q=0.1$. The two inner target radii are $c_{1}=0.15 \mathrm{~m}$ and $c_{2}=0.075 \mathrm{~m}$. For simplicity, we consider a coil switching function $f_{S}(t)$ that represents the current being turned on in some switching time $\tau_{S}$. This is accomplished by setting $f_{S}=0$ for $t<0$ and $f_{S}=1$ for all $t>\tau_{S}$. In order that $f_{S}^{\prime \prime}(t)$ should be continuous, we choose $f_{S}(t)$ to be a cubic spline function in the interval $0<t<\tau_{S}$.

To begin, we consider an $x$-gradient coil, so that in polar coordinates, the two inner target fields are

$$
H_{T Z}^{(1)}=H_{\max } \cos \theta, \quad H_{T Z}^{(2)}=H_{\max }\left(c_{2} / c_{1}\right) \cos \theta .
$$

Figure 2 shows the winding pattern on the primary coil, for an $x$-gradient coil designed with regularization parameter $\lambda_{1}=10^{17}$. This value was chosen after careful experimentation, and represents about the optimum trade-off between being able to reproduce the target fields (14) faithfully on the one hand, and reducing the acoustic noise on the other. The large value of this parameter is necessary to compensate for the very small value of the constant $\Delta_{0}$ in equation (5) that determines the size of the coil deflections. The two smoothing parameters were chosen to be $\lambda_{P}=\lambda_{S}=10^{-12}$. The dashed lines in the figure indicate windings with the current reversed. In Figure 2, the azimuthal angle $\theta$ is plotted on the horizontal axis, so that the diagram may be considered to be wrapped around the surface of the cylinder $r=a$. Winding patterns on the shield at $r=b$ are similar to those in Figure 2 and so are not shown here, although the current direction is opposed so as to cancel the fields external to the coil.

The deflection caused to the coil by Lorentz forces is shown in Figure 3, for the design illustrated in Figure 2. For ease of visibility, the deflections have been multiplied by $10^{4}$, so that the coil shape shown is actually the surface $r=a+10^{4} u_{R}$ for some time $t>\tau_{S}$. Clearly the greatest deflections 


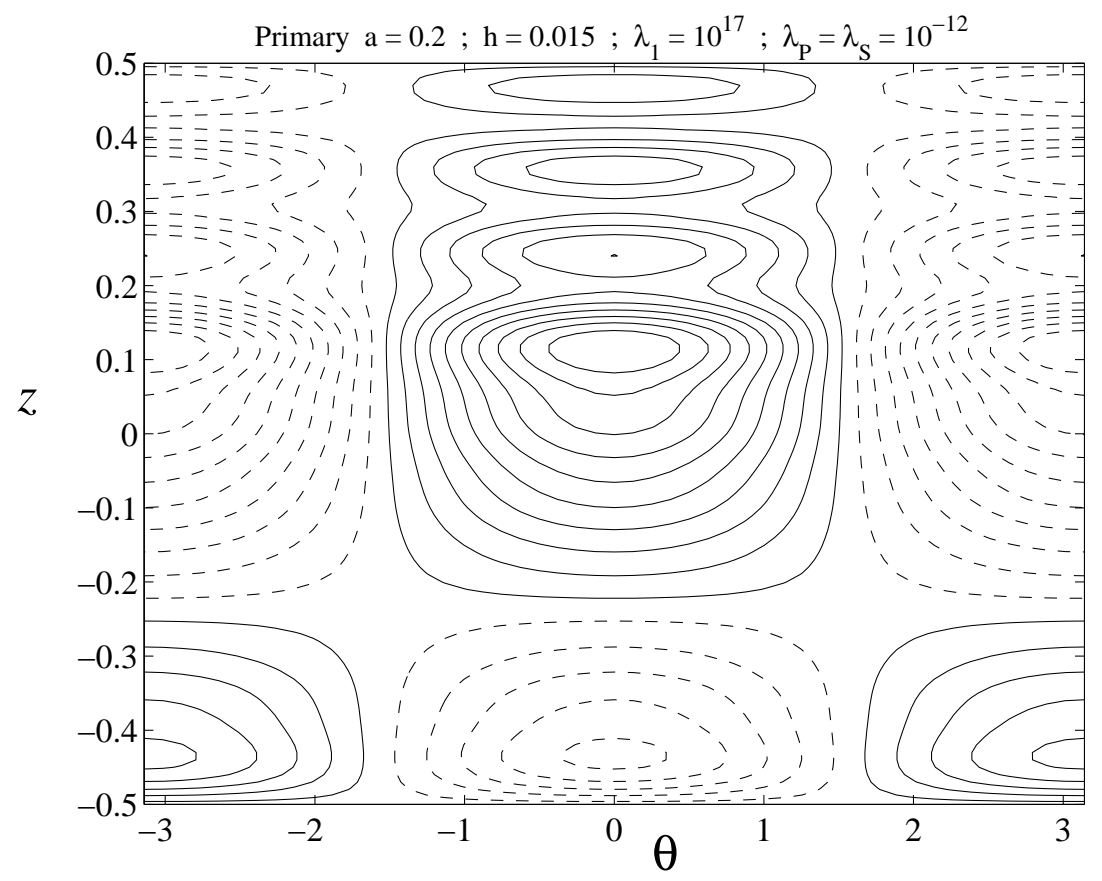

FiguRE 2: Winding pattern for an $x$-gradient coil of thickness $h=15 \mathrm{~mm}$, for the primary at $r=a$. Dashed lines indicate reversed windings. 


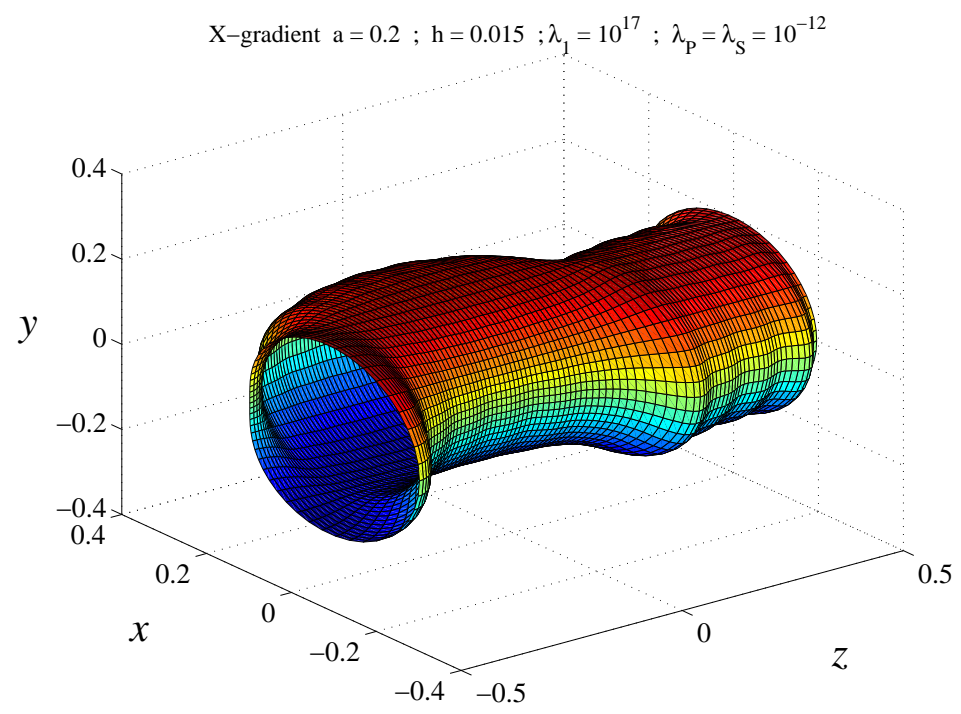

Figure 3: The coil shape in response to Lorentz forces, for the $x$-gradient coil in Figure 2. Displacements have been magnified by a factor of 10,000 for ease of viewing. 


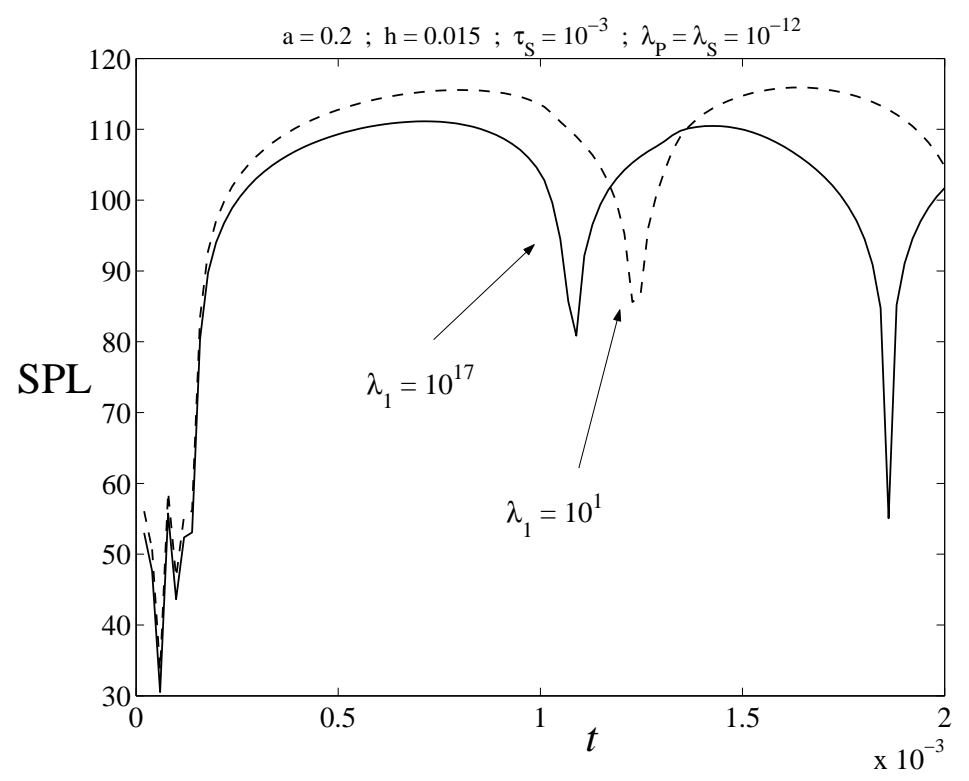

Figure 4: Sound pressure level (in decibels) as a function of time, for an un-optimized coil (dashed line) and the optimized coil in Figure 2 (solid line). The coil thickness is $15 \mathrm{~mm}$ and the switching time is $1 \mathrm{~ms}$.

occur in the asymmetrically located target region (at the left end of the coil), as a result of which the coil undergoes an overall lateral displacement towards the negative $x$-direction. Smaller deflections also occur at the end of the coil further from the target region, but these appear to be more circularly symmetric.

Figure 4 gives a comparison between the noise levels produced by two different coils designed with regularizing parameters $\lambda_{1}=10$ and $\lambda_{1}=10^{17}$. The first of these is effectively not optimized with respect to noise level, whereas the second case, illustrated in Figures 2 and 3, represents optimal conditions. Sound levels were computed using equation (8), and are shown at the point $(r, \theta, z)=\left(c_{1}, 0,(p+q) L / 2\right)$ at the mid-point on the outer surface of the inner target regions shown in Figure 1. In each case, the switching 
time is $\tau_{S}=10^{-3} \mathrm{~s}(1 \mathrm{~ms})$. Time $t$ is shown on the horizontal axis, and the vertical scale gives the sound pressure level in decibels. The dashed line shows the noise history of the un-optimized coil and indicates a peak level of $116 \mathrm{~dB}$. The solid line corresponds to the acoustic noise produced by the optimized coil, and shows peak noise levels of $111 \mathrm{~dB}$. Thus the optimized coil of Figures 2 and 3 results in a reduction of $5 \mathrm{~dB}$ in overall noise level.

We conclude this section with a brief discussion of a $z$-gradient coil, for which the two inner target fields have the forms

$$
H_{T Z}^{(1)}=H_{T Z}^{(2)}=\frac{2 H_{\max }}{(q-p)}\left[\frac{z}{L}-\frac{p+q}{2}\right] .
$$

These fields (15) have similarly been used to design coils optimized for noise, although space is insufficient here to discuss these in detail. For these coils, the winding patterns consist of circular loops of wire around the cylinder, on planes orthogonal to the coil axis.

We illustrate the deflection caused by a $z$-gradient coil, optimized for minimum noise production, in Figure 5. The displacements are again magnified by a factor of $10^{4}$ for ease of viewing, so that the surfaces shown represent graphs of $r=a+10^{4} u_{R}$. However, unlike the $x$-gradient coil in Figure 3, the coil here retains circular symmetry as the windings are simply arranged circularly around the cylinder.

\section{Conclusion}

A method has been presented for computing the deflection of a cylindrical gradient coil due to Lorentz forces, and estimating its noise production. Tikhonov regularization helps design coils that reduce noise by minimizing the coil deflection. Nevertheless, it is typically found that only about $5 \mathrm{~dB}$ noise reduction is achieved by these designs (recalling, however, that the noise 


\section{Shielded Z-gradient coil $\lambda_{1}=10^{17} ; c_{3}=1.1 \mathrm{~b}$}

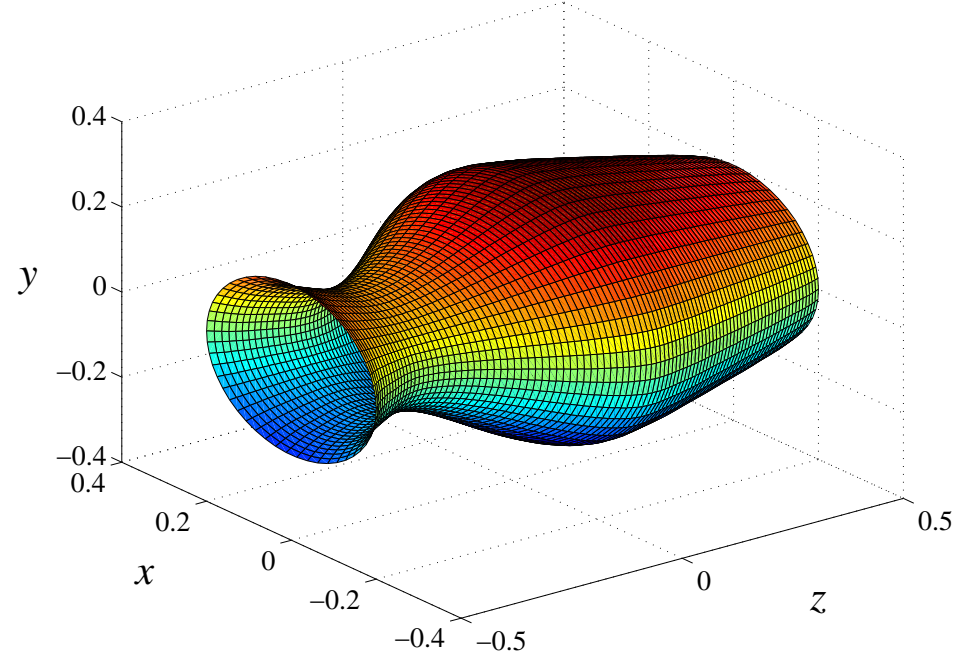

Figure 5: The coil shape in response to Lorentz forces, for a noise optimized $z$-gradient coil. Displacements have been magnified by a factor of 10,000 for ease of viewing. 
scale in Equation (8) is logarithmic). It is possible to reduce noise further by increasing the coil thickness $h$, as expected, although this is ultimately limited by practical design considerations. Our analysis assumed that the coil deflection is mainly radial, although the results, such as in Figure 3, suggest that other displacement modes might also usefully be considered. This is left for future work.

Acknowledgements: This work is supported in part by ARC grant number DP0343350.

\section{References}

[1] A. P. Boresi and K. P. Chong. Elasticity in engineering mechanics. Elsevier, New York 1987. C22

[2] M. A. Brideson, L. K. Forbes and S. Crozier. Determining complicated winding patterns for shim coils using stream functions and the target-field method. Concepts Magn. Reson., 14:9-18, 2002. doi:10.1002/cmr.10000 C22

[3] B. L. W. Chapman and P. Mansfield. A quiet gradient-coil set employing optimized, force-shielded, distributed coil designs. J. Magn. Reson. B, 107:152-157, 1995. C19

[4] S. Crozier and D. M. Doddrell. Gradient-coil design by simulated annealing. J. Magn. Reson. A, 103:354-357, 1993. C19

[5] L. M. Delves and J. L. Mohamed. Computational Methods for Integral Equations. Cambridge University Press, Cambridge, 1985. C19

[6] L. K. Forbes and S. Crozier. A novel target-field method for finite-length magnetic resonance shim coils: Part 1: Zonal shims. $J$. 
Phys. D: Appl. Phys., 34:3447-3455, 2001.

doi:10.1088/0022-3727/34/24/305 C19

[7] L. K. Forbes and S. Crozier. A novel target-field method for finite-length magnetic resonance shim coils: Part 2: Tesseral shims. $J$. Phys. D: Appl. Phys., 35:839-849, 2002. doi:10.1088/0022-3727/35/9/303 C19

[8] L. K. Forbes and S. Crozier. A novel target-field method for magnetic resonance shim coils: Part 3: Shielded zonal and tesseral coils. J. Phys. D: Appl. Phys., 36:68-80, 2003. doi:10.1088/0022-3727/36/2/302 C19

[9] E. M. Haacke, R. W. Brown, M. R. Thompson and R. Venkatesan. Magnetic Resonance Imaging: Physical principles and sequence design. Wiley, New York, 1999. C18, C21

[10] J. Jin. Electromagnetic Analysis and Design in Magnetic Resonance Imaging. Biomedical Engineering Series, CRC Press, Boca Raton, 1999. C18

[11] P. Mansfield, B. Haywood and R. Coxon. Active acoustic control in gradient coils for MRI. Magn. Reson. Med., 46:807-818, 2001. C19

[12] C. K. Mechefske, G. Yao, W. Li, C. Gazdzinski and B. K. Rutt. Modal analysis and acoustic noise characterization of a $4 \mathrm{~T}$ MRI gradient coil insert. Concepts Magn. Reson. B, 22:37-49, 2004. doi:10.1002/cmr.b.20013 C19

[13] W. Shao and C. K. Mechefske. Acoustic analysis of a gradient coil winding in an MRI scanner. Concepts Magn. Reson. B, 24:15-27, 2005. doi:10.1002/cmr.b.20023 C19

[14] W. Shao and C. K. Mechefske. Analysis of the sound field in finite length infinite baffled cylindrical ducts with vibrating walls of finite impedance. J. Acoust. Soc. Am., 117:1728-1736, 2005. C19 
[15] R. Turner. A target field approach to optimal coil design. J. Phys D: Appl. Phys., 19:147-151, 1986. C18, C19

[16] M. T. Vlaadingerbroeck and J. A. den Boer. Magnetic Resonance Imaging: Theory and practice, 3rd edition. Springer Verlag, Berlin, 2003. C18 


\section{Author addresses}

1. L. K. Forbes, School of Mathematics \& Physics, University of Tasmania, Private Bag 37, Hobart, Tasmania 7001, Australia. mailto:Larry . Forbes@utas . edu . au

2. M. A. Brideson, School of Mathematics \& Physics, University of Tasmania, Private Bag 37, Hobart, Tasmania 7001, Australia.

3. S. Crozier, School of Information Technology \& Electrical Engineering, University of Queensland, St. Lucia, Queensland 4072, Australia.

mailto:stuart@itee.uq.edu.au

4. P. T. While, School of Mathematics \& Physics, University of Tasmania, Private Bag 37, Hobart, Tasmania 7001, Australia. 